\title{
Community hospitals in Oxfordshire: their effect on the use of specialist inpatient services
}

\author{
JAMES E BAKER, ${ }^{1}$ MICHAEL GOLDACRE, ${ }^{1}$ AND J A MUIR GRAY' \\ From the University Department of Community Medicine and General Practice, ${ }^{1}$ and the District Department of \\ Community Medicine, Oxfordshire Health Authority, ${ }^{2}$ Radcliffe Infirmary, Oxford OX2 $6 \mathrm{HE}$
}

SUMMARY About one-third of the general practices in the Oxfordshire Health District have access to beds in community hospitals as well as district general hospitals. Hospital Activity Analysis data were used to calculate the average number of hospital beds occupied daily by patients registered with each general practice in the district. Practices with and without access to community hospitals were compared to determine whether such access was associated with (i) a reduction in the use of beds in general medical, geriatric, and other specialties, and (ii) an increase in overall utilisation rates. The rate of use of general medical and geriatric beds in district general hospitals by practice populations with access to community hospitals was about half that of populations without such access. Utilisation rates overall, combining the use of beds in both district general hospitals and community hospitals, were a little higher in populations with access to both community hospitals and district general hospitals than in those with access to district general hospitals only.

At the time of this study there were 10 community hospitals in the Oxfordshire Health District. They ranged in size from 9 to 79 beds and together had a total of 315 beds. Patients may be admitted to these hospitals if their general practitioner has a contract with the health authority for this purpose.

Community hospitals are regarded as a primary care facility in the Oxford Region. It is envisaged that patients will be admitted to community hospital rather than to district general hospital beds if hospital care under the supervision of a general practitioner is appropriate. ${ }^{12}$ Patients may also be discharged to community hospitals from a district general hospital following lengths of stay which might be longer without the community hospital option. Thus it is expected that the provision of beds in community hospitals will reduce the use of beds in district general hospitals.

With the exception of $x$-ray facilities at some hospitals, the provision of diagnostic services differs little from that available for the investigation of patients in their own homes or in general practitioners' surgeries. The hospitals are not used for the treatment of children or for surgical operations but they do provide convalescent care after surgery in the district general hospital. Admission policies specifically exclude patients with predominantly psychiatric problems. Three of the hospitals have a small complement of maternity beds which are not considered in this paper. Thus the effect of the two mechanisms by which community hospital beds may reduce use of district general hospital inpatient care (reduction in the number and duration of admissions) would be expected to be greatest for general medical and geriatric specialties. Any effect on the amount of care provided by other specialties would be expected to be produced mainly by the second of the two mechanisms (transfer of patients) and to be smaller.

It has also been suggested, however, that the provision of community hospitals may result in an increase in the total amount of inpatient care used by the populations served. ${ }^{3}$

The analyses reported here were undertaken to determine whether general practice populations with access to community hospitals do, in fact, have lower than average utilisation rates of district general hospitals; and whether such populations have higher than average overall utilisation rates.

\section{Method}

Hospital Activity Analysis covers all hospital admissions in the Oxfordshire Health District (population 503000 ) and it includes a record of the practice with which each inpatient is registered. 
These data were used to compare hospitalisation rates between groups of general practices in the years 1979-81.

General practices were included in the analysis if (a) the Oxfordshire Family Practitioner Committee was the responsible Committee for the practice, and (b) the practice looked to hospitals in Oxfordshire for district general hospital inpatient services. Hospital admissions recorded in Hospital Activity Analysis in surrounding districts were checked to ensure that practices met the second criterion.

Sixty seven practices, with 498000 registered patients, met the criteria for inclusion. The number of patients registered with each practice in the years covered by the study was obtained from the Oxfordshire Family Practitioner Committee with the consent of the Local Medical Committee. Practice population figures were supplied for patients under 65 years of age, 65-74 years, and 75 years and over. Further subdivision of these populations by age group was not available.

The 67 practices were grouped, and the results are presented, as follows:

Group 1: practices in Oxford city, none of which had access to community hospitals;

Group 2: other practices, in the small towns and rural areas of the District, without access to community hospitals;

Group 3: other practices, in the small towns and rural areas, with access to community hospitals.

The average number of hospital beds occupied per day were calculated, and are expressed per 10000 people in each group of practices, for specialties as follows:

(a) patients (including long stay cases) in district general hospital beds under the care of general physicians and geriatricians. (These specialties are not considered separately because the designation of consultant care between the specialties is not wholly separate in this district;)

(b) patients in district general hospital beds under the care of other specialists, excluding obstetricians and psychiatrists;

(c) patients in community hospitals in general practitioner medical beds.

The average number of beds used per day per 10000 people was used as an index of rate of use of inpatient care in preference to admission rate, the only other index which could have been calculated from routinely available data. The latter is only one determinant of amount of inpatient care, the other being duration of hospitalisation. Admission rate could be a misleading index of amount of use of inpatient care in situations where a policy of discharge for weekends exists or the practice is to admit patients frequently for brief periods rather than less frequently and for longer periods.

\section{Results}

Table 1 shows the average number of people registered per annum in each group of practices. Table 2 shows the average number of beds occupied daily per 10000 people registered in each group of practices. Considering all specialties together, age standardised bed use rates by populations with community hospital access were about $2 \%$ higher than the rates for Oxford city practices and about $6 \%$ higher than those for other practices without community hospital access. Bed use rates in general medicine and geriatrics for people aged 65 and over in populations with community hospital access were about half those found in populations without such access. For people under 65 years of age the bed use rate in these specialties in populations with community hospital access was about three-quarters that of the populations without access. Bed use rates in other district general hospital specialties for

Table 1 Average number of people registered per annum with each group of practices (thousands)

\begin{tabular}{lcccc}
\hline \multicolumn{5}{c}{ Age group } \\
\cline { 2 - 5 } Practice group * $^{4}$ & $<65$ & $65-74$ & $75+$ & All ages \\
\hline 1 & 155.0 & 15.1 & 9.8 & 179.9 \\
2 & 95.9 & 8.6 & 5.8 & 110.3 \\
3 & 181.2 & 16.2 & 10.3 & 207.9 \\
\hline
\end{tabular}

* Group 1 =practices in Oxford city;

Group 2 =other practices without access to community hospitals;

Group 3=practices with access to community hospitals.

Table 2 Average number of beds used daily per 10000 people by specialty, general practice, and age group

\begin{tabular}{|c|c|c|c|c|}
\hline \multirow[b]{2}{*}{ Specialty } & \multicolumn{4}{|c|}{ General practice group } \\
\hline & Age group & Group 1 & Group 2 & Group 3 \\
\hline $\begin{array}{l}\text { General medicine } \\
\text { and geriatrics }\end{array}$ & $\begin{array}{l}<65 \\
65-74 \\
75+ \\
\text { Total }\end{array}$ & $\begin{array}{r}1.78 \\
29.71 \\
134.76 \\
10.99\end{array}$ & $\begin{array}{r}1.86 \\
25.79 \\
112.93 \\
9.56\end{array}$ & $\begin{array}{r}1 \cdot 36 \\
13 \cdot 81 \\
51 \cdot 75 \\
4.98\end{array}$ \\
\hline $\begin{array}{l}\text { General practitioner } \\
\text { beds (community hospitals) }\end{array}$ & $\begin{array}{l}<65 \\
65-74 \\
75+ \\
\text { Total }\end{array}$ & $\begin{array}{l}- \\
- \\
-\end{array}$ & $\begin{array}{l}- \\
- \\
-\end{array}$ & $\begin{array}{r}0 \cdot 84 \\
18 \cdot 10 \\
94 \cdot 85 \\
7 \cdot 13\end{array}$ \\
\hline Other specialties & $\begin{array}{l}<65 \\
65-74 \\
75+ \\
\text { Total }\end{array}$ & $\begin{array}{r}9 \cdot 14 \\
29 \cdot 55 \\
37 \cdot 85 \\
12 \cdot 27\end{array}$ & $\begin{array}{r}9.58 \\
26.49 \\
40.26 \\
12.53\end{array}$ & $\begin{array}{r}9.31 \\
22.90 \\
29.00 \\
11.42\end{array}$ \\
\hline All specialties & $\begin{array}{l}<65 \\
65-74 \\
75+ \\
\text { Total }\end{array}$ & $\begin{array}{r}10.92 \\
59.26 \\
172.61 \\
23.26\end{array}$ & $\begin{array}{r}11.44 \\
52.28 \\
153.19 \\
22.09\end{array}$ & $\begin{array}{r}11 \cdot 51 \\
54 \cdot 81 \\
175.60 \\
23.53\end{array}$ \\
\hline
\end{tabular}

'Total' rates are age-standardised by the direct method. The whole study populations in each age category were used to weight the age specific rates. 
patients aged 65 years and over were a little lower in populations with community hospital access than in those without. The differences between these populations were much less striking than those found in general medicine and geriatrics.

The highest rate of use of general practitioner beds in the community hospitals was found for patients in the oldest age group, and it was in this age group that the most striking deficit of use of beds in the district general hospitals was found. We considered the possibility that the difference in rates shown in table 2 was attributable to a small number of practices with highly atypical rates. The figure shows the distribution of bed use rates, for individual practices, in general medicine and geriatrics for people aged 65-74 and 75 years and over. While there is considerable variation between individual practices in bed use rates, the figure shows a general tendency

(Age 65-74 years)

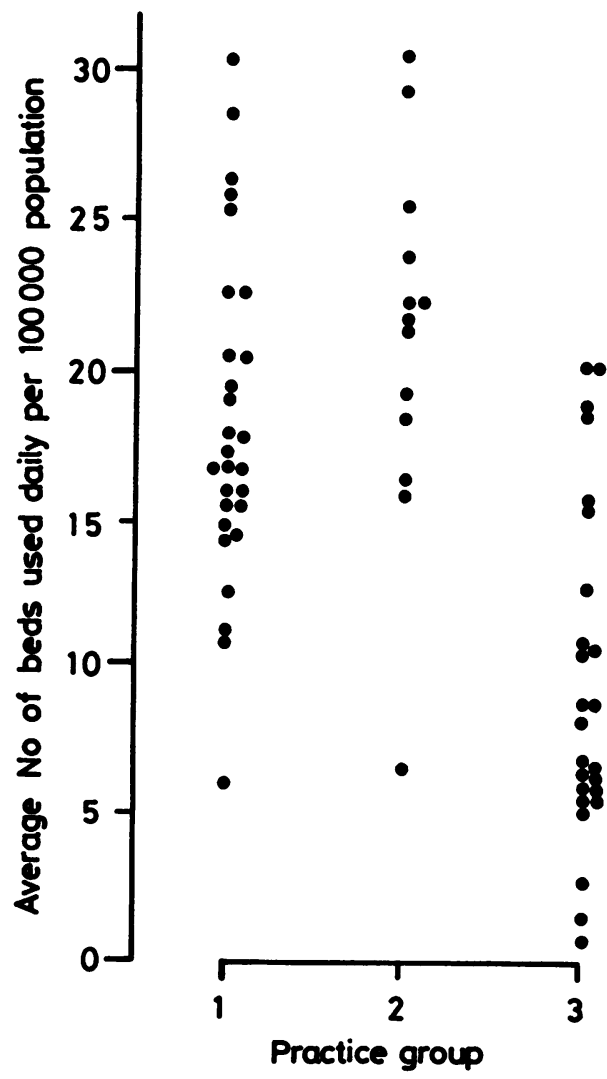

for rates in these age groups and specialties to be lower in practices with community hospital access than in others.

In Oxfordshire patients are admitted to both geriatric wards and community hospitals for indefinite (long term) periods of care. The analyses were repeated after excluding patients with lengths of stay of one year or more, and the results differed very little from those already given. This indicates that small numbers of patients with very long lengths of stay did not contribute substantially to the findings of this study.

\section{Discussion}

This analysis indicates that the use of community hospital beds was associated with relatively low rates of use of district general hospital beds, notably for the

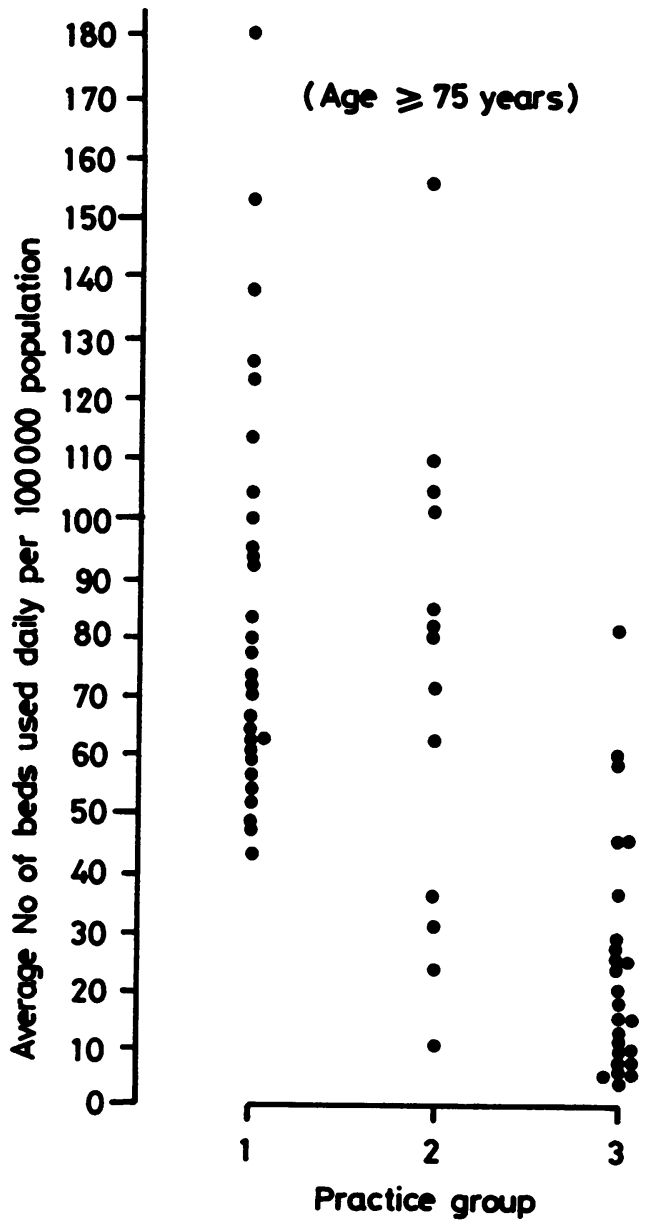

Average number of beds used daily per 10000 population: people aged 65-74 years and 75 years and over (each plot represents one practice). 
care of the elderly in general medicine and geriatrics, in this district during the study period.

The possibility exists that access to community hospitals has been provided for populations with lower than average needs for district general hospital inpatient care. However, to support this explanation one would have to postulate that needs were low mainly for that very section of the population which makes most use of community hospital care (elderly patients with medical conditions). Furthermore, the community hospitals and the practice populations with access to them are not clustered together in a discrete part of the district: they are widely scattered across it. The most likely explanation for our findings is that community hospitals have indeed been used as a substitute for district general hospital care and that, in this district, the provision of community hospitals has reduced the use of district general hospital care by the populations served by them.

It has been suggested ${ }^{3}$ that provision of community hospitals in Oxfordshire may lead to admission of patients who would not otherwise gain access to National Health Service hospital beds. The total utilisation rates in community and other hospitals for all specialties (table 2) are higher for each age category of the population with community hospital access than for the two other population groups. This could imply that provision of community hospitals has led to admission and/or longer hospitalisation of patients who could have been cared for at home. An alternative explanation is that some people in the population without community hospital access would have been better managed in hospital but were cared for at home because their general practitioner did not have access to a community hospital. This would have implications for community services were it the case. A third possibility is that more use of private institutional care is made by the populations without community hospital access: the health authority makes use of private hospital beds for convalescence of patients (often for two week periods) following care in the district general hospital, and preliminary investigation indicates that the majority of patients so placed do not have access to a community hospital.

Calculation of the costs and benefits of providing community hospital beds as an alternative to district general hospital beds is complex and beyond the scope of this study. However, assuming that the differences in bed use rates between the types of practice in table 2 are wholly or mainly attributable to the presence or absence of community hospitals, certain broad conclusions can be drawn. The rate of use of general medical and geriatric beds in district general hospitals by practice populations with access to community hospitals was about half that of populations without such access. This represented a reduction in bed use of about 5 beds per 10000 population served per day. There was an additional reduction of about 1 bed per 10000 population per day in the use of beds in other specialties. On the other hand, the overall utilisation rates of combined use of district general hospital and community hospital beds were a little higher in the populations with access to community hospitals than in populations with access to district general hospitals alone. This represents a difference in use of about 1 bed per 10000 population per day.

Other studies, of different design, have suggested that the provision of peripheral health care facilities reduces the demand on central services in Oxfordshire. ${ }^{4}$ The observed trade-off between the use of peripheral general practitioner community hospitals, which have limited diagnostic and therapeutic facilities, and the use of large central hospitals with facilities for providing specialist care emphasises that community hospitals may have animportant place in supporting provision of primary care and complementing services of specialist hospitals in the Oxfordshire District. One cannot, of $\underline{\underline{O}} \leq$ course, assume that this pattern will invariably be ${ }^{-} \vec{c}$ found elsewhere. The need for inpatient care in NHS hospitals may vary with the level of morbidity in the population being served, differences in the behaviourc. $\vec{\theta}$ of carers and patients and variations in provision and? accessibility of hospital and other forms of care. However, where data about inpatients' general practitioners are recorded in routine hospital statistics, other districts can undertake studies similar to ours as a preliminary step in assessing the use made of their local hospitals.

\section{References}

${ }^{1}$ Oxford Regional Hospital Board. The work of a cottage hospital in a rural community. Oxford Regional Hospital Board 1965.

'Oxford Regional Health Authority. Regional strategy 1984-1994. Oxford Regional Health Authority 1984.

${ }^{3}$ Cullington DE, Schofield C. Contribution of the local health authority. In: Bennett AE, ed. Community hospitals: Progress in development and evaluation. Oxford Regional Hospital Board 1974; chapter 4.

Loudon ISL. The general practitioner and the hospital. In: Fry J, ed. Trends in general practice. London: The Royal College of General Practitioners, 1977; 83-110.

${ }^{5}$ Goldacre MJ, Gatherer A. Peripheral outpatient clinics: use, costs and benefits. Br J Prev Soc Med 1977; 31: 205-8. 